
\title{
25 Research Soure \\ The elevated risk of sight-threatening cataract in diabetes with retinopathy: a cohort study
}

\section{Chan-Wei Nien}

Show Chwan Memorial Hospital

\section{Chia-Yi Lee}

Show Chwan Memorial Hospital

\section{Hung-Chi Chen}

Chang Gung Medical Foundation

\section{Shih-Chun Chao}

Show Chwan Memorial Hospital

\section{Hung-Jui Hsu}

Show Chwan Memorial Hospital

\section{Shih-Hao Tzeng}

Show Chwan Memorial Hospital

\section{Jing-Yang Huang}

Chung Shan Medical University

\section{Hsin-Fong Miao}

Jen-Teh Junior College of Medicine Nursing and Management

Hung-Yu Lin

Show Chwan Memorial Hospital

Shun-Fa Yang ( $\square$ ysf@csmu.edu.tw)

Chung Shan Medical University https://orcid.org/0000-0002-0365-7927

\section{Research article}

Keywords: diabetes mellitus, diabetic retinopathy, cataract surgery, epidemiology

Posted Date: January 16th, 2020

DOl: https://doi.org/10.21203/rs.2.21037/v1

License: (9) This work is licensed under a Creative Commons Attribution 4.0 International License. Read Full License 


\section{Abstract}

Background To survey the effect of diabetic retinopathy (DR) on the development of sight-threatening cataract via National Health Insurance Research Database of Taiwan.

Methods Patients diagnosed with diabetes mellitus (DM) and DR were enrolled in the study group. The age and gender-matched DM individuals without DR and patients without DM served as the DM control group and non-DM control group, both with 1:4 ratios. The outcome was set as the performance of cataract surgery. Cox proportional hazard regression was used to calculate the adjusted hazard ratio (aHR) of DR with considering multiple factors of cataract formation.

Results A total of 3,297 DR patients, 13,188 DM control patients and 13,188 non-DM controls were enrolled. There were 919 events (27.87 percent) of sight-threatening cataract in the study group while another 1,108 events (8.40 percent) in the DM control group and 957 events ( 7.26 percent) in the non-DM control group. After multivariable analysis, the study group showed a higher aHR of cataract surgery $(2.93,95 \% \mathrm{Cl}: 2.60-3.30)$ and a higher cumulative probability of cataract surgery compared to both the DM control and non-DM control groups (both Log rank $P<0.001)$. Besides, both the proliferative DR (3.90, 95\% Cl: $3.42-4.45)$ and non-proliferative DR $(2.35,95 \% \mathrm{Cl}$ : 2.08-2.65) subgroups showed a higher aHR of cataract surgery than the DM control group.

Conclusion The presence of DR will increase the risk for development of sight-threatening cataract that surgery is warranted, and the effect is prominent in both the proliferative DR and non-proliferative DR.

\section{Background}

Cataract is the leading cause of reversible blindness throughout the world.[1, 2] In a study conducted in Asian and Oceania recently, the prevalence of cataract-induced blindness and moderate-to-severe visual impairment in general population is more than 35 percent.[3] The associated ocular morbidities of cataract include glaucoma and uveitis while both of them can also result in impaired vision.[4,5] Surgical management is always indicated for sight-threatening cataract but some complications may occur after surgery like postoperative endophthalmitis and cystoid macular edema.[6, 7]

About the possible risk factors for the formation of cataract, ultraviolet exposure has shown a significant association to the cataract development.[8] In addition, trans pars plana vitrectomy would alter the oxygen concentration in vitreous cavity and lead to following cataract.[9, 10] Both the systemic and local steroid administration would cause the cataract,[11, 12] while certain medications including phenothiazines, amiodarone, statin and metformin may also increase the possibility of cataract formation.[13-16] Diabetes mellitus (DM) is a metabolic disorder featuring with hyperglycemia and insulin dysregulation which influence eye mainly on the retina and cataract development.[17-21] The risk of cataract formation can be up to five-folds in patients with DM compared to non-DM induviduals.[22] 
Although the relationship between DM and cataract occurrence has been well-established before, there are still some issues to be addressed. Whether the duration of DM was a risk factor of developing cataract showed conflicting results in the previous studies.[18, 23] In addition, the presence of diabetic retinopathy (DR), which indicates a worse ocular condition in DM patients,[19, 24] may also influence the incidence of cataract but has rarely been reported elsewhere. Moreover, the DR can be categorized into non-proliferative diabetic retinopathy (NPDR) and a more severe proliferative diabetic retinopathy (PDR), [25] while the effect of different DR severity on cataract formation that needs surgery is also unknown.

Herein, we aimed to evaluate the effect of DR, including both NPDR and PDR, on the developing of cataract that surgical intervention is indicated to improve the vision using the National Health Insurance Research Database (NHIRD) of Taiwan. In addition, the duration of DM and other possible potential risk factors of sight-threatening cataract will also be surveyed in the multivariate model.

\section{Material And Method}

\section{Data Source}

The retrospective population-based cohort study was conducted in accordance with the Declaration of Helsinki, and was approved by both the National Health Insurance Administration and the Institutional Review Board of Chung Shan Medical University (Identification code: CS-17075). Supported by the Taiwan National Health Research Institutes, the NHIRD contains medical information of insurance claims from nearly all the population in Taiwan. Those claims data were obtained from the Longitudinal Health Insurance Database 2005 version (LHID) in the current study. The LHID includes information on two million individuals which randomly selected from the NHIRD documents for the year 2005. The LHID were connected from 1 January 2000, until the 31 December 2016, and the International Classification of Diseases, Ninth Revision (ICD-9) as well as International Classification of Diseases, Tenth Revision (ICD10) were applied for disease diagnosis and identification. Information on the medications prescribed for the patients and the demographic data, socioeconomic condition, and living region of the patients are also accessible from the NHIRD.

\section{Patients Selection}

All the subjects included in the current study were selected in 2005 and the relevant data of participants were traced from 2000 to 2016. Patients were regarded as a case with DM if their medical documents demonstrated (1) a history of DM, (2) the arrangement of blood glucose test, glycosylated hemoglobin test, or oral glucose tolerance test before the diagnosis of DM, and (3) the DM was diagnosed by an internal medicine or family medicine department. To more accurately elucidate the association between DM and related retinopathy and cataract, the following exclusion criteria were applied to exclude certain impaired ocular conditions: (1) diagnosed with of legal blindness before the diagnosis of DM; (2) the arrangement of any type of eyeball removal surgery before the diagnosis of DM; (3) receipt a diagnosis of ophthalmic tumors before the diagnosis of DM; (4) receipt a diagnosis of prominent ocular trauma at any time; (5) the arrangement of cataract surgery, diagnosis of pseudophakia, and Nd: YAG capsulotomy 
before the index date; (6) diagnosed with myopia since myopia is associated with cataract formation in previous experience;[26] (7) diagnosed with DR before the diagnosis of DM; (8) the event is before the index date; and (9) receipt of a diagnosis of DM before 2005 to exclude patients with extremely long DM disease period. After the exclusion, the study group was set as the individuals with a diagnosis of DR. Besides, every subject in the study group was age and gender-matched with four DM patients that without DR and non-DM individuals, which served as the DM control group and non-DM control group respectively. Patients with DR who could not be matched with either four DM patients without DR or four non-DM patients were excluded and the same exclusion criteria applied to the study group was also used before matching in both the DM control and non-DM control groups. The index date was regarded as the date of DR diagnosis in the study group and the same day was also set as the index date in matched DM control group and non-DM control group.

\section{Main Outcome Measurement}

The development of sight threatening cataract was regarded as the primary outcome in the current study which was based on the emergence of senile/complicated/diabetic cataract-related diagnostic codes plus the receipt of cataract surgery after the index date. Those cataract-related diagnostic codes that indicate clearly underlying etiology were not included in the current study to prevent confusion as well as overestimation. In addition, only those subjects that received those diagnostic codes by an ophthalmologist were recognized as having achieved the outcome and were included in the study.

\section{Demographic data and Co-Morbidities}

To make the health status of each subject more homogenous, we also surveyed the influences of age, gender and the following systemic diseases in the multivariable analysis: hypertension, ischemic heart diseases, hyperlipidemia, congestive heart failure, cerebrovascular disease, dementia, chronic pulmonary disease, rheumatoid arthritis and osteoarthritis, kidney disease, liver cirrhosis, alcoholic liver disease, gout:, atopic dermatitis, and allergic otolaryngologic diseases. Similarly, dry eye diseases (DED), uveitis, glaucoma, age-related macular degeneration (AMD) and the arrangement of trans pars plana vitrectomy (TPPV) were considered in the multivariate model to standardize the ocular condition. Since certain medications may lead to the development of cataract, the following medications were also enrolled in our analysis: systemic steroid including prednisolone, methylprednisolone, hydrocortisone, triamcinolone, dexamethasone, topical steroid including prednisolone, fluorometholone, betamethasone, triamcinolone, dexamethasone, phenothiazines, amiodarone, statin including rosuvastatin, atorvastatin, simvastatin, pravastatin, lovastatin, fluvastatin and pitavastatin, and metformin. We traced the data in the NHIRD longitudinally from the index date of each participant to (1) the date of sight-threatening cataract development, (2) withdrawal from the National Health Insurance program, or (3) the time of 31 December 2016.

\section{Statistical Analysis}

SAS version 9.4 (SAS Institute Inc, NC, USA) was applied for all the statistical analyses. After age and gender-matching at 1:4 ratio of the study group, DM-control group and non-DM control groups, the 
Poisson regression was conducted to calculate the incidence rate, crude relative risk and related $95 \%$ confidence intervals $(\mathrm{Cl})$. We conducted the Cox proportional hazard regression to calculate the adjusted hazard ratios (aHR) of sight-threatening cataract among the three groups by incorporating the aforementioned demographic information, systemic comorbidities, ocular diseases, medications and disease interval in the multivariate analysis. To investigate the effect of DR severity on the development of sight-threatening cataract, the study group was further divided into NPDR subgroup and PDR subgroup in the multivariate model and the aHR of each subgroup was analyzed. Moreover, the sensitivity analysis with aHR of sight-threatening cataract which stratified by the age, gender, severity of DR and DM interval (lesser than two years, two to five years and more than five years) were performed for the subgroup analysis. Also, we drew Kaplan-Meier curves to present the cumulative probability of sight-threatening cataract between the study, DM control and non-DM control groups. After that, we used the log rank test to evaluate the significance among all the three survival curves. Since almost all the individuals enrolled in the NHIRD belong to Han/Taiwanese population, ethnicity was thus not considered as a possible confounding factor. Statistical significance was set at $\mathrm{P}<0.05$.

\section{Results}

A total of 3,297 DR patients, 13,188 DM control patients and 13,188 non-DM controls were enrolled in the current study after selection as shown in Fig. 1. Due to the matching process, the age and gender distributions were identical among the three groups. For the potential risk factors, significant difference about the ratio of co-morbidities and medications was found among groups except dementia, chronic pulmonary disease, rheumatoid arthritis as well as osteoarthritis and atopic dermatitis (Table 1). 
Table 1

Basic characteristics among the study and control groups.

\begin{tabular}{|c|c|c|c|c|c|}
\hline Characteristics & $\begin{array}{l}\text { Non-DM } \\
\text { Control } \\
n=13188\end{array}$ & $\begin{array}{l}\text { DM Control } \\
n=13188\end{array}$ & $\begin{array}{l}\text { DR } \\
n=3297\end{array}$ & P1 & P2 \\
\hline Age & & & & 1.0000 & 1.0000 \\
\hline$<40$ & $672(5.1 \%)$ & $672(5.1 \%)$ & $168(5.1 \%)$ & & \\
\hline $40-59$ & $7168(54.35 \%)$ & $7168(54.35 \%)$ & $1792(54.35 \%)$ & & \\
\hline $60-79$ & $5048(38.28 \%)$ & $5048(38.28 \%)$ & $1262(38.28 \%)$ & & \\
\hline$>=80$ & $300(2.27 \%)$ & $300(2.27 \%)$ & $75(2.27 \%)$ & & \\
\hline Gender & & & & 1.0000 & 1.0000 \\
\hline Male & $6872(52.11 \%)$ & $6872(52.11 \%)$ & $1718(52.11 \%)$ & & \\
\hline Female & $6316(47.89 \%)$ & $6316(47.89 \%)$ & $1579(47.89 \%)$ & & \\
\hline \multicolumn{6}{|l|}{ Co-morbidities } \\
\hline Hypertension & $4441(33.67 \%)$ & $8696(65.94 \%)$ & $2321(70.4 \%)$ & $<.0001 *$ & <.0001* \\
\hline Ischemic heart diseases & $1573(11.93 \%)$ & $2977(22.57 \%)$ & $653(19.81 \%)$ & $<.0001 *$ & $0.0006 *$ \\
\hline Hyperlipidemia & $3425(25.97 \%)$ & $8751(66.36 \%)$ & $2134(64.73 \%)$ & $<.0001 *$ & 0.0771 \\
\hline Congestive heart failure & $567(4.3 \%)$ & $1260(9.55 \%)$ & $324(9.83 \%)$ & $<.0001 *$ & 0.6343 \\
\hline Cerebrovascular disease & $1255(9.52 \%)$ & 2135(16.19\%) & $530(16.08 \%)$ & $<.0001 *$ & 0.8739 \\
\hline Dementia & $193(1.46 \%)$ & $262(1.99 \%)$ & $35(1.06 \%)$ & 0.0772 & $0.0004^{*}$ \\
\hline $\begin{array}{l}\text { Chronic pulmonary } \\
\text { diseases }\end{array}$ & $2736(20.75 \%)$ & $3763(28.53 \%)$ & $715(21.69 \%)$ & 0.2353 & <. $0001 *$ \\
\hline $\begin{array}{l}\text { Rheumatoid arthritis as } \\
\text { well as osteoarthritis }\end{array}$ & $367(2.78 \%)$ & $442(3.35 \%)$ & $102(3.09 \%)$ & 0.3369 & 0.4586 \\
\hline Kidney disease & $695(5.27 \%)$ & $1507(11.43 \%)$ & $493(14.95 \%)$ & $<.0001 *$ & <. $0001 *$ \\
\hline $\begin{array}{l}\text { Hepatitis and liver } \\
\text { cirrhosis }\end{array}$ & $3080(23.35 \%)$ & $5511(41.79 \%)$ & $1032(31.3 \%)$ & $<.0001 *$ & $<.0001 *$ \\
\hline Alcoholic liver disease & $125(0.95 \%)$ & $401(3.04 \%)$ & $82(2.49 \%)$ & $<.0001 *$ & 0.0919 \\
\hline
\end{tabular}




\begin{tabular}{|c|c|c|c|c|c|}
\hline Characteristics & $\begin{array}{l}\text { Non-DM } \\
\text { Control } \\
n=13188\end{array}$ & $\begin{array}{l}\text { DM Control } \\
n=13188\end{array}$ & $\begin{array}{l}\text { DR } \\
n=3297\end{array}$ & P1 & $\mathrm{P} 2$ \\
\hline Gout & $1642(12.45 \%)$ & $3079(23.35 \%)$ & $547(16.59 \%)$ & $<.0001 *$ & $\begin{array}{l}<.0001 * \\
0\end{array}$ \\
\hline Atopic dermatitis & $475(3.6 \%)$ & $667(5.06 \%)$ & $133(4.03 \%)$ & 0.2389 & $0.0144 *$ \\
\hline $\begin{array}{l}\text { Allergic otolaryngologic } \\
\text { diseases }\end{array}$ & $2705(20.51 \%)$ & $3067(23.26 \%)$ & $563(17.08 \%)$ & $<.0001 *$ & $\begin{array}{l}<.0001 * \\
0.0\end{array}$ \\
\hline DED & 1093(8.29\%) & $1241(9.41 \%)$ & $466(14.13 \%)$ & $<.0001 *$ & $\begin{array}{l}<.0001 * \\
0.0\end{array}$ \\
\hline Uveitis & $111(0.84 \%)$ & $122(0.93 \%)$ & $139(4.22 \%)$ & <. $0.001 *$ & <.0001* \\
\hline Glaucoma & $297(2.25 \%)$ & $376(2.85 \%)$ & $270(8.19 \%)$ & $<.0001 *$ & <. $0001 *$ \\
\hline AMD & $130(0.99 \%)$ & $157(1.19 \%)$ & $172(5.22 \%)$ & $<.0001 *$ & <.0001* \\
\hline $\begin{array}{l}\text { Retinal diseases warrant } \\
\text { trans pars plana } \\
\text { vitrectomy }\end{array}$ & $19(0.14 \%)$ & $11(0.08 \%)$ & $181(5.49 \%)$ & $<.0001 *$ & $<.0001 *$ \\
\hline \multicolumn{6}{|l|}{ Co-medication } \\
\hline Systemic steroid & $1974(14.97 \%)$ & $2676(20.29 \%)$ & $752(22.81 \%)$ & $<.0001 *$ & $0.0014 *$ \\
\hline Topical steroid & $1737(13.17 \%)$ & $2048(15.53 \%)$ & $1810(54.9 \%)$ & $<.0001 *$ & <. $0001 *$ \\
\hline Phenothiazines & $385(2.92 \%)$ & $671(5.09 \%)$ & $236(7.16 \%)$ & $<.0001 *$ & $\stackrel{<}{0.0001 *}$ \\
\hline Amiodarone & $54(0.41 \%)$ & $136(1.03 \%)$ & $26(0.79 \%)$ & $0.0051^{\star}$ & 0.2065 \\
\hline Statin & $993(7.53 \%)$ & $4069(30.85 \%)$ & $1348(40.89 \%)$ & $<.0001 *$ & $\begin{array}{l}<.0001 * \\
\end{array}$ \\
\hline Metformin & $397(3.01 \%)$ & 6558(49.73\%) & $2510(76.13 \%)$ & $<.0001 *$ & <. $0001 *$ \\
\hline Duration of DM (years) & & & & 1.0000 & 1.0000 \\
\hline 0 & 13188(100\%) & $0(0 \%)$ & $0(0 \%)$ & & \\
\hline$<2$ & $0(0 \%)$ & $6456(48.95 \%)$ & $1614(48.95 \%)$ & & \\
\hline $2-5$ & $0(0 \%)$ & $3828(29.03 \%)$ & $957(29.03 \%)$ & & \\
\hline$\geq 5$ & $0(0 \%)$ & $2904(22.02 \%)$ & $726(22.02 \%)$ & & \\
\hline
\end{tabular}




\begin{tabular}{|c|c|c|c|c|c|}
\hline Characteristics & $\begin{array}{l}\text { Non-DM } \\
\text { Control } \\
n=13188\end{array}$ & $\begin{array}{l}\text { DM Control } \\
n=13188\end{array}$ & $\begin{array}{l}\text { DR } \\
n=3297\end{array}$ & P1 & P2 \\
\hline \multicolumn{4}{|l|}{ Type of DR } & - & - \\
\hline Without DR & $13188(100 \%)$ & $13188(100 \%)$ & $0(0 \%)$ & & \\
\hline NPDR & $0(0 \%)$ & $0(0 \%)$ & $1788(54.2$ & & \\
\hline PDR & $0(0 \%)$ & $0(0 \%)$ & $1509(45$. & & \\
\hline \multicolumn{6}{|c|}{ P1: comparison between non-diabetes mellitus control group and the diabetic retinopathy group } \\
\hline \multicolumn{6}{|c|}{ P2: comparison between diabetes mellitus control group and the diabetic retinopathy group } \\
\hline \multicolumn{6}{|c|}{ DED: dry eye disease } \\
\hline \multicolumn{6}{|c|}{ AMD: age-related macular degeneration } \\
\hline \multicolumn{6}{|c|}{ DM: diabetes mellitus } \\
\hline \multicolumn{6}{|c|}{ DR: diabetic retinopathy } \\
\hline \multicolumn{6}{|c|}{ NPDR: non-proliferative diabetic retinopathy } \\
\hline \multicolumn{6}{|c|}{ PDR: proliferative diabetic retinopathy } \\
\hline * denotes signif & & & & & \\
\hline
\end{tabular}

During the whole follow-up period, there were 919 events (27.87 percent) of sight-threatening cataract in the study group whose crude relative risk was higher compared to the 1,108 events (8.40 percent) in the DM control group and 957 events (7.26 percent) in the non-DM control group (crude relative risk: 4.62, $95 \% \mathrm{Cl}: 4.22-5.06$, Table 2). After the multivariate analysis considering the demographic data, systemic diseases, prominent ocular diseases and medications that may lead to sight-threatening cataract, the study group still showed a significant higher aHR compared to the non-DM control group $(2.93,95 \% \mathrm{Cl}$ : 2.60-3.30). However, the DM control group did not reveal a higher aHR of cataract surgery performance compared to the non-DM control group $(1.02,95 \% \mathrm{Cl}: 0.92-1.12)$. The other risk factors of sightthreatening cataract include age from 60 years to 79 years (aHR: 3.50, 95\% Cl: 3.22-3.81), age older than 80 years, hypertension (aHR:1.25, 95\% Cl: 1.14-1.36), ischemic heart disease (aHR: $1.12,95 \% \mathrm{Cl}$ : $1.02-$ 1.22), chronic pulmonary disease (aHR: $1.11,95 \% \mathrm{Cl}: 1.02-1.21$ ), rheumatoid arthritis as well as osteoarthritis (aHR: 1.23, 95\% Cl: 1.03-1.47), kidney disease (aHR: $1.22,95 \% \mathrm{Cl}: 1.09-1.36)$, gout (aHR: 1.16, 95\% Cl: 1.06-1.27), atopic dermatitis (aHR: 1.19, 95\% Cl: 1.01-1.40), DED (aHR: 1.16, 95\% Cl: 1.041.28), uveitis (aHR: 1.34, 95\% Cl: 1.06-1.70), glaucoma (aHR: 1.42, 95\% Cl: 1.23-1.65), AMD (aHR: 1.32, 95\% Cl: 1.12-1.59), retinal diseases received TPPV (aHR: 1.79, 95\% Cl: 1.48-2.24), systemic steroid (aHR: 1.16, 95\% Cl: 1.06-1.26) and topical steroid (aHR: 1.89, 95\% Cl: 1.75-2.06) (Table 3). Furthermore, the Kaplan-Meier curves revealed a significant higher cumulative probability of sight-threatening 
cataract compared to both the DM control (Log rank $\mathrm{P}<0.001)$ and non-DM control groups (Log rank $\mathrm{P}<$ 0.001), while the cumulative probability between the DM control group and non-DM control groups were similar (Log rank $\mathrm{P}>0.05$, Fig. 2).

Table 2

Incidence of sight-threatening cataract among the study and control groups.

\begin{tabular}{|llll|}
\hline Events & $\begin{array}{l}\text { Non-DM control } \\
\mathbf{n = 1 3 1 8 8}\end{array}$ & $\begin{array}{l}\text { DM control } \\
\mathbf{n = 1 3 1 8 8}\end{array}$ & $\begin{array}{l}\text { DR } \\
\mathbf{n}=\mathbf{3 2 9 7}\end{array}$ \\
\hline Follow up person months & 680920 & 656839 & 142148 \\
\hline New cataract case & 957 & 1108 & 919 \\
\hline Incidence rate $(95 \% \mathrm{Cl})$ & $1.41(1.32-1.50)$ & $1.69(1.59-1.79)$ & $6.47(6.06-6.90)$ \\
\hline Crude relative risk $(95 \% \mathrm{Cl})$ & Reference & $1.20(1.10-1.31) *$ & $4.62(4.22-5.06) *$ \\
\hline DM: diabetes mellitus & & & \\
\hline DR: diabetic retinopathy & & & \\
\hline \# per 1000 person months & & & \\
\hline Cl: confidential interval & & & \\
\hline * denotes significant difference & & \\
\hline
\end{tabular}


Table 3

Multiple Cox proportional hazard regression for estimation of adjusted hazard ratios on sight-threatening cataract.

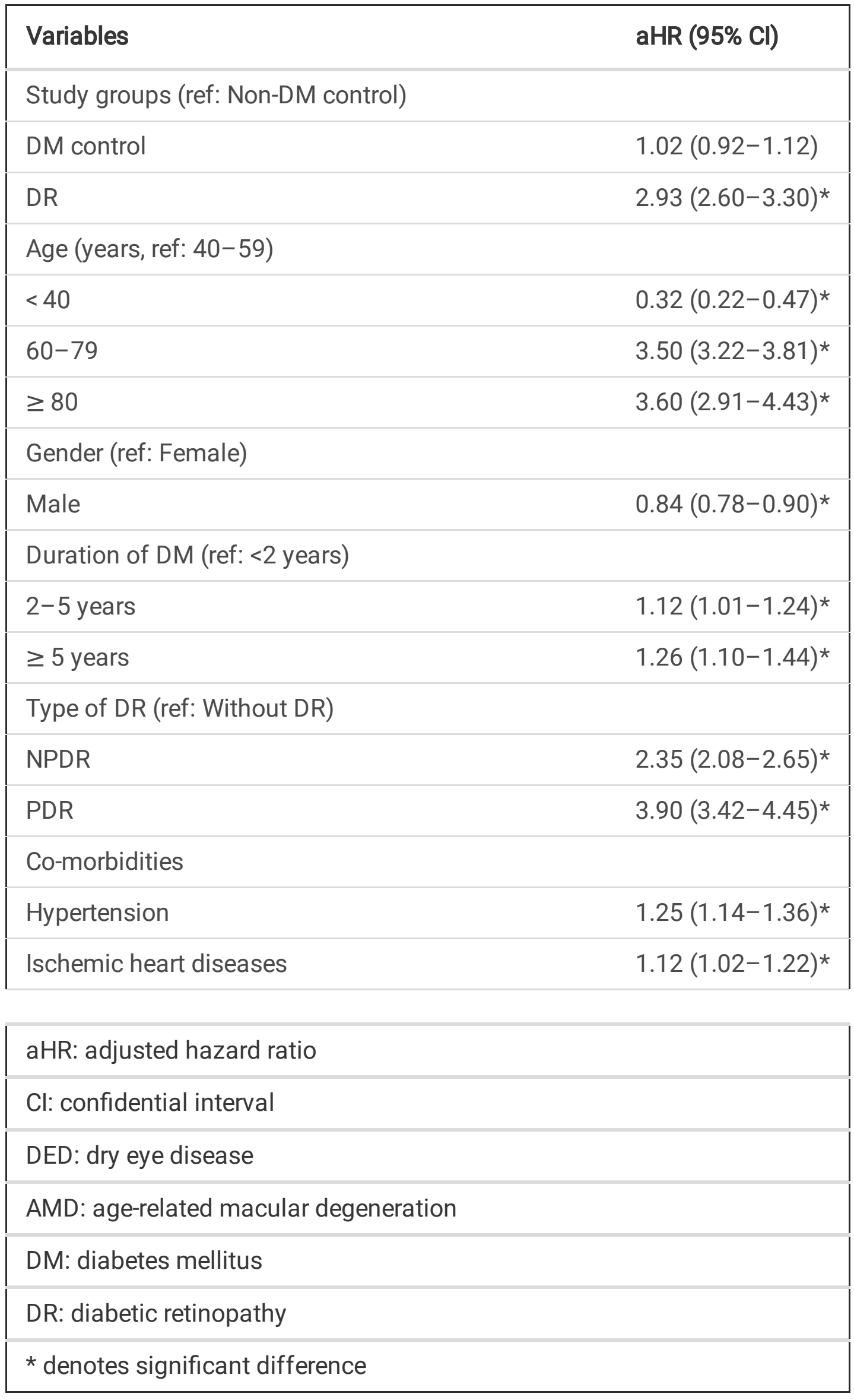




\begin{tabular}{|ll|}
\hline Variables & aHR $(95 \% \mathrm{Cl})$ \\
\hline Hyperlipidemia & $1.01(0.93-1.10)$ \\
\hline Congestive heart failure & $1.12(1.00-1.27)$ \\
\hline Cerebrovascular disease & $1.04(0.94-1.14)$ \\
\hline Dementia & $0.70(0.53-0.92)$ \\
\hline Chronic pulmonary diseases & $1.11(1.02-1.21)^{\star}$ \\
\hline Rheumatoid arthritis as well as osteoarthritis & $1.23(1.03-1.47)^{\star}$ \\
\hline Kidney disease & $1.22(1.09-1.36)^{\star}$ \\
\hline Hepatitis and liver cirrhosis & $0.96(0.88-1.04)$ \\
\hline Alcoholic liver disease & $1.00(0.74-1.34)$ \\
\hline Gout & $1.16(1.06-1.27)^{\star}$ \\
\hline Atopic dermatitis & $1.19(1.01-1.40)^{\star}$ \\
\hline Allergic otolaryngologic diseases & $0.97(0.89-1.07)$ \\
\hline DED & $1.16(1.04-1.28)^{\star}$ \\
\hline Uveitis & $1.34(1.06-1.70)^{\star}$ \\
\hline Glaucoma & $1.42(1.23-1.65)^{\star}$ \\
\hline AMD & $1.32(1.12-1.59)^{\star}$ \\
\hline Retinal diseases warrant trans pars plana vitrectomy & $1.79(1.48-2.24)^{\star}$ \\
\hline Co-medication & \\
\hline Systemic steroid & \\
\hline Topical steroid & \\
\hline AHR: adjusted hazard ratio & \\
\hline Cl: confidential interval & \\
\hline DED: dry eye disease & \\
\hline AMD: age-related macular degeneration & \\
\hline DR: diabetes mellitus & $1.89(1.75-2.06)^{\star}$ \\
\hline
\end{tabular}




\begin{tabular}{|ll|}
\hline Variables & aHR (95\% Cl) \\
\hline Phenothiazines & $1.03(0.88-1.20)$ \\
\hline Amiodarone & $1.23(0.87-1.74)$ \\
\hline Statin & $1.03(0.94-1.12)$ \\
\hline Metformin & $1.06(0.97-1.16)$ \\
\hline aHR: adjusted hazard ratio & \\
\hline Cl: confidential interval & \\
\hline DED: dry eye disease & \\
\hline AMD: age-related macular degeneration & \\
\hline DM: diabetes mellitus & \\
\hline DR: diabetic retinopathy & \\
\hline * denotes significant difference & \\
\hline
\end{tabular}

In the subgroup analysis of DM population, the PDR subgroup showed a significantly higher aHR of cataract surgery performance $(3.90,95 \% \mathrm{Cl}: 3.42-4.45)$ than the DM control group, and the rate of cataract surgery was also higher in the NPDR subgroup than the DM control group (aHR: $2.35,95 \% \mathrm{Cl}$ : 2.08-2.65) (Table 3). On the other hand, the rates of sight-threatening cataract in both the PDR and NPDR subgroups were significantly higher compared to the DM control group whether considering different disease intervals of DM and genders (Table 4). Nevertheless, the NPDR patients younger than 40 years old and the PDR subjects older than 80 years old did not showed a significantly higher aHR of sight-threatening cataract compared to the DM control group (Table 4). Besides, the PDR subgroup revealed a numerically higher aHR than NPDR subgroup if stratified by duration of DM, gender and age groups except the elderly that aged older than 80 years (Table 4). 
Table 4

The sensitivity analysis for the adjusted hazard ratio stratified by duration of diabetes mellitus, gender and age groups.

\begin{tabular}{|c|c|c|c|c|c|}
\hline \multirow[t]{2}{*}{ Subgroups } & \multicolumn{3}{|c|}{ Incidence rate* $(95 \% \mathrm{Cl})$ of Cataract } & \multirow{2}{*}{$\begin{array}{l}\text { aHR1 }(95 \% \\
\text { Cl) }\end{array}$} & \multirow[t]{2}{*}{ aHR2 (95\% Cl) } \\
\hline & DM control & NPDR & PDR & & \\
\hline \multicolumn{6}{|c|}{$\begin{array}{l}\text { Duration of DM } \\
\text { (Year) }\end{array}$} \\
\hline$<2$ & $\begin{array}{l}1.47(1.36- \\
1.60)\end{array}$ & $\begin{array}{l}3.79(3.26- \\
4.41)\end{array}$ & $\begin{array}{l}7.76(6.93- \\
8.69)\end{array}$ & $\begin{array}{l}2.20(1.83- \\
2.63)^{\star}\end{array}$ & $\begin{array}{l}4.06(3.39- \\
4.88)^{\star}\end{array}$ \\
\hline $2-5$ & $\begin{array}{l}1.89(1.7- \\
2.09)\end{array}$ & $\begin{array}{l}5.15(4.36- \\
6.08)\end{array}$ & $\begin{array}{l}9.63(8.15- \\
11.38)\end{array}$ & $\begin{array}{l}2.21(1.79- \\
2.73)^{\star}\end{array}$ & $\begin{array}{l}3.46(2.71- \\
4.41)^{\star}\end{array}$ \\
\hline$\geq 5$ & $\begin{array}{l}2.29 \\
2.65)\end{array}$ & $\begin{array}{l}8.04(6.56- \\
9.87)\end{array}$ & $\begin{array}{l}13.74(11.04- \\
17.11)\end{array}$ & $\begin{array}{l}3.07(2.33- \\
4.03)^{\star}\end{array}$ & $\begin{array}{l}4.14(2.98- \\
5.76)^{\star}\end{array}$ \\
\hline $\begin{array}{l}\text { P for } \\
\text { interaction }\end{array}$ & 0.3526 & & & & \\
\hline \multicolumn{6}{|l|}{$\begin{array}{l}\text { Gender } \\
\text { subgroups }\end{array}$} \\
\hline Male & $\begin{array}{l}1.35(1.23- \\
1.47)\end{array}$ & $\begin{array}{l}4.36(3.75- \\
5.06)\end{array}$ & $\begin{array}{l}8.30(7.37- \\
9.34)\end{array}$ & $\begin{array}{l}2.70(2.24- \\
3.25)^{*}\end{array}$ & $\begin{array}{l}4.00(3.29- \\
4.86)^{*}\end{array}$ \\
\hline Female & $\begin{array}{l}2.05(1.9- \\
2.21)\end{array}$ & $\begin{array}{l}5.23(4.6- \\
5.95)\end{array}$ & $\begin{array}{l}9.48(8.36- \\
10.75)\end{array}$ & $\begin{array}{l}2.12(1.81- \\
2.49)^{\star}\end{array}$ & $\begin{array}{l}3.81(3.18- \\
4.57)^{\star}\end{array}$ \\
\hline $\begin{array}{l}\text { P for } \\
\text { interaction }\end{array}$ & 0.0618 & & & & \\
\hline \multicolumn{6}{|l|}{$\begin{array}{l}\text { Age at index } \\
\text { date }\end{array}$} \\
\hline$<40$ & $\begin{array}{l}0.22(0.11- \\
0.43)\end{array}$ & $\begin{array}{l}0.22(0.03- \\
1.54)\end{array}$ & $\begin{array}{l}3.96(2.46- \\
6.37)\end{array}$ & $\begin{array}{l}1.51(0.17- \\
13.26)\end{array}$ & $\begin{array}{l}16.99(3.61- \\
80.01)^{\star}\end{array}$ \\
\hline $40-59$ & $\begin{array}{l}0.73(0.65- \\
0.82)\end{array}$ & $\begin{array}{l}2.49(2.07- \\
2.99)\end{array}$ & $\begin{array}{l}7.57(6.75- \\
8.48)\end{array}$ & $\begin{array}{l}2.85(2.26- \\
3.58)^{\star}\end{array}$ & $\begin{array}{l}5.96(4.79- \\
7.41)^{\star}\end{array}$ \\
\hline $60-79$ & $\begin{array}{l}3.60(3.36- \\
3.86)\end{array}$ & $\begin{array}{l}8.48(7.53- \\
9.57)\end{array}$ & $\begin{array}{l}13.41(11.65- \\
15.43)\end{array}$ & $\begin{array}{l}2.16(1.86- \\
2.51)^{\star}\end{array}$ & $\begin{array}{l}2.72(2.25- \\
3.28)^{\star}\end{array}$ \\
\hline$\geq 80$ & $\begin{array}{l}3.13(2.21- \\
4.43)\end{array}$ & $\begin{array}{l}12.75(8.22- \\
19.76)\end{array}$ & $\begin{array}{l}12.53(6.74- \\
23.29)\end{array}$ & $\begin{array}{l}2.96(1.51- \\
5.81)^{\star}\end{array}$ & $\begin{array}{l}2.30(0.95- \\
5.61)\end{array}$ \\
\hline $\begin{array}{l}\text { P for } \\
\text { interaction }\end{array}$ & $<0.0001$ & & & & \\
\hline \multicolumn{6}{|c|}{ *Incidence rate, per 1000 person months } \\
\hline DM: diabet & & & & & \\
\hline
\end{tabular}




\section{DM control NPDR PDR}

Cl)

NPDR: non-proliferative diabetic retinopathy

PDR: proliferative diabetic retinopathy

aHR1: non-proliferative diabetic retinopathy versus diabetes mellitus control

aHR2: proliferative diabetic retinopathy versus diabetes mellitus control

aHR: adjusted hazard ratio

Cl: confidential interval

* denotes significant difference

\section{Discussion}

Briefly, the current study illustrate a significant correlation of sight-threatening cataract and patients with DR compared to those with solitary DM or without DM, with an elevated risk of about three-folds after adjusting multiple potential risk factors. In addition, both the the patients with PDR and NPDR showed significantly higher possibility of developing sight-threatening cataract than subjects with DM but without DR. Other possible risk factors of sight-threatening cataract include mainly older age, cardiovascular diseases, chronic pulmonary disease, inflammatory diseases, ocular disorders and use of steroid. The major pathophysiology of DM to induced cataract is thought to be the fluid accumulation process.[27] The hyperglycemic status leads to the additional production of sorbital from the Polyol pathway for glucose metabolism in lens.[18] Due to elevated osmotic pressure caused by the build-up of sorbital,[27] the excess fluid influx the lens which lead to cell swelling, membrane impairment and following cataract. [18] On the other hand, the DR would lead to an intraocular condition of hypoxia, ischemia and inflammation.[24] In the previous review article concerning PDR, the reactive oxygen spices, inflammatory cytokines and vascular endothelial growth factors were increased in the vitreous cavity,[28] and the oxidative stress is also the risk factors of cataract formation according to preceding laboratory studies. [29-31] As a consequence, it is possible that the DR would influence the microenvironment in eye more than solitary DM and result in cataract development, which supported by the result of the current study.

Several researches have evaluated the association of DM and cataract and unanimously indicate a significant risk of cataract in patients with DM. In a previous review article written by Drinkwater et al, the age and blood glucose level are correlated to a higher risk of cataract formation.[23] However, another study revealed the duration of DM is the major risk factor for the occurrence of cataract.[18] In the current study, the study group showed a significantly higher cumulative probability (both Log-rank $P<0.001$ ) compared to the DM control group and non-DM control group after adjusting multiple potential risk factors for cataract formation including the age and disease duration of DM. According to our awareness, 
this is a preliminary experience to illustrate the presence of DR will significantly elevated the risk of sightthreatening cataract compared to those with DM but without ocular manifestations. About the time sequence in the current study, only the cataract formation after the index date was accounted for the outcome achievement thus the causal relationship is guaranteed. Moreover, individuals with pre-existing cataract, receiving previous cataract surgery or receiving Nd:YAG laser capsulotomy to retard the posterior capsular opacification after cataract surgery were excluded from the current study, and we only accounted those with senile/complicated/diabetic cataract that need surgery. Accordingly, the effect of DR on the occurrence of sight-threatening cataract is relative accuracy compared to previous studies which enrolled patients with baseline cataract.[32] About the follow up period, the mean disease interval of DM in the current study was about 10 years, which was above the average compared to the previous studies.[23] Thus, the significant correlation between DR and sight-threatening cataract in the current study may with lesser bias.

In the multivariate analysis concerning PDR, NPDR and DM control groups, individuals with PDR and NPDR revealed a significantly higher aHR of developing sight-threatening cataract compared to DM control group, while the aHR in PDR subgroup was also numerically higher than the NPDR subgroup. Since the PDR are with a higher level of oxidative stress compared to NPDR,[28] it is reasonable that the occurrence of sight-threatening cataract in PDR subgroup is numerically higher than the NPDR subgroup because of the elevation of risk factor. Nevertheless, the aHR of sight-threatening cataract was significantly elevated in the NPDR group compared to the control group which implied that the existence of DR is a major risk factor of cataract development whether in non-proliferative or in proliferative form after considering multiple potential risk factors. For the subgroup analysis stratified by age, gender and duration of DM, the PDR still illustrated the highest aHR in nearly all the subgroup, which further strength the influence of PDR on sight-threatening cataract. Interestingly, the aHR of PDR was extremely higher than the NPDR and DM control subgroups in the population aged younger than 40 years old. The possible explanation is that young patients with PDR usually experience more severe DR and a higher rate of blindness as demonstrated in previous study.[33]

For the epidemiology aspect, DM influences about 5.7 percent of global population,[34] while the prevalence increases to 10.3 percent in the one Asian population.[35] Among those individuals with DM, the gross prevalence of DR is about 24.7 percent in a similar population.[36] Similarly, cataract is the major etiology of legal blindness in the world which account for about 30-50 percent of the severe visual impairment.[3] The occurrence rate of sight-threatening cataract in the study group is approximately 27.9 percent which was three-folds higher than the 8.4 percent in the DM control group and the 7.3 percent in the non-DM control group. In a previous population-based study conducted in the same region, the prevalence of self-reported cataract was about 11.8 percent in 2013,[37] which was much lower than the study group in the current study. Since both DM and cataract affect a majority of population and the DR will increase the risk of sight-threatening cataract with a gross population, strictly blood sugar control and periodically ophthalmic examination in patients with DM is advocated. 
About the risk factors of developing sight-threatening cataract other than DR, all the ophthalmic diseases enrolled in the multivariate analysis were correlated to the occurrence of sight-threatening cataract. The glaucoma, uveitis and receipt of TPPV are known risk factors of cataract formation, $[4,5,9]$ and the advanced age and inflammatory reaction in both DED and AMD may result in the higher cataract development.[38, 39] The cardiovascular diseases and inflammatory diseases are the two groups of systemic diseases that associated with a higher cataract occurrence, and the exact pathophysiology need further investigation. Both the systemic steroid and topical steroid are correlated to the existence of sightthreatening cataract, which was also proven in previous studies.[11, 12] Age is also a well-established risk factor for cataract formation,[40] and the elevated aHR in individuals with older age in all the groups of the current study further supports this concept.

Certain limitations still existed in the current study. First, the retrospective study design and the use of claimed data will restricted the accuracy and standardization in the current study. Second, the degree of ultraviolet exposure cannot be assess by the NHIRD thus may lead to certain bias. In addition, the blood glucose level is absent in the NHIRD and the severity of DM is unknown despite we enrolled several complications of severe DM in the multivariate analysis involving ischemic heart disease, cerebrovascular disease and kidney disease. Besides, the demand of cataract surgery is relative subjective so the severity of cataract would not be the same in the study population. However, the photopsia caused by posterior subcapsular cataract and the irregular lenticular astigmatism could impair the vision despite good visual acuity. Accordingly, all the patients need cataract surgery may experience visual impairment at some degrees that affect their daily activity.

\section{Conclusion}

In conclusion, the present of DR is a significant risk factor for the development of sight-threatening cataract that need surgery to retain the visual acuity after adjusting multiple potential risk factors. Furthermore, individuals with both PDR and NPDR are at a higher risk of sight-threatening cataract compared to patients with DM but without DR. On the other hand, the rate of sight-threatening cataract is similar between patients with DM but without DR and individuals without DM. Further large scale prospective study to survey the effect of treating DR on the development of sight-threatening cataract is mandatory.

\section{Declarations}

\section{Ethics approval and consent to participate}

The study adhered to the declaration of Helsinki in 1964 and its late amendment. Specifically, the current study was approved by both the National Health Insurance Administration and the Institutional Review Board of Chung Shan Medical University, Taichung, Taiwan. In addition, the need of informed consents was waived by both the Institutional Review Board of Chung Shan Medical University, Taichung, Taiwan. 


\section{Consent for publication}

Not applicable.

\section{Availability of data and materials}

Since the data involved in the current study is locked in certain institutions by the National Health Insurance Administration in which only the results can be taken out, it cannot be shared as a resource.

\section{Competing interests}

The authors declare that they have no competing interests.

\section{Funding}

No.

\section{Authors' contributions}

CWN, CYL, HYL and YSF contributed to the concept and study design. The data was collected and analyzed by SCC, HJH, SHT and JYH. Then CYL, HCC and HFM drafted the manuscript. All the authors including CWN, CYL, HCC, SCC, HJH, SHT, JYH, HFM, HYL and YSF were involved in the critical revision of the manuscript, supervision of the manuscript and final approval of the submission.

\section{Acknowledgement}

Not applicable

\section{References}

1. Lee CM, Afshari NA: The global state of cataract blindness. Current opinion in ophthalmology 2017, 28(1):98-103.

2. Olson RJ, Mamalis N, Werner L, Apple DJ: Cataract treatment in the beginning of the 21st century. American journal of ophthalmology 2003, 136(1):146-154.

3. Keeffe JE, Casson RJ, Pesudovs K, Taylor HR, Cicinelli MV, Das A, Flaxman SR, Jonas JB, Kempen $\mathrm{JH}$, Leasher $\mathrm{J}$ et al: Prevalence and causes of vision loss in South-east Asia and Oceania in 2015: magnitude, temporal trends and projections. The British journal of ophthalmology 2018.

4. Chan NS, Ti SE, Chee SP: Decision-making and management of uveitic cataract. Indian journal of ophthalmology 2017, 65(12):1329-1339.

5. Ellant JP, Obstbaum SA: Lens-induced glaucoma. Documenta ophthalmologica Advances in ophthalmology 1992, 81(3):317-338. 
6. Wielders LH, Lambermont VA, Schouten JS, van den Biggelaar FJ, Worthy G, Simons RW, Winkens B, Nuijts RM: Prevention of Cystoid Macular Edema After Cataract Surgery in Nondiabetic and Diabetic Patients: A Systematic Review and Meta-Analysis. American journal of ophthalmology 2015, 160(5):968-981.e933.

7. Garg P, Roy A, Sharma S: Endophthalmitis after cataract surgery: epidemiology, risk factors, and evidence on protection. Current opinion in ophthalmology 2017, 28(1):67-72.

8. Miyashita H, Hatsusaka N, Shibuya E, Mita N, Yamazaki M, Shibata T, Ishida H, Ukai Y, Kubo E, Sasaki $\mathrm{H}$ : Association between ultraviolet radiation exposure dose and cataract in Han people living in China and Taiwan: A cross-sectional study. PloS one 2019, 14(4):e0215338.

9. Keyal K, Liao X, Liu G, Yang S, Wang F: Post-vitrectomy cataract acceleration in phakic eyes: a review. Discovery medicine 2017, 24(134):305-311.

10. Ji Y, Rong X, Lu Y: Metabolic characterization of human aqueous humor in the cataract progression after pars plana vitrectomy. BMC ophthalmology 2018, 18(1):63.

11. James ER: The etiology of steroid cataract. Journal of ocular pharmacology and therapeutics : the official journal of the Association for Ocular Pharmacology and Therapeutics 2007, 23(5):403-420.

12. Kiernan DF, Mieler WF: The use of intraocular corticosteroids. Expert opinion on pharmacotherapy 2009, 10(15):2511-2525.

13. Pietrowska K, Dmuchowska DA, Krasnicki P, Bujalska A, Samczuk P, Parfieniuk E, Kowalczyk T, Wojnar M, Mariak Z, Kretowski A et al: An exploratory LC-MS-based metabolomics study reveals differences in aqueous humor composition between diabetic and non-diabetic patients with cataract. Electrophoresis 2018, 39(9-10):1233-1240.

14. Ikaheimo $\mathrm{K}$, Kettunen $\mathrm{R}$, Mantyjarvi M: Visual functions and adverse ocular effects in patients with amiodarone medication. Acta ophthalmologica Scandinavica 2002, 80(1):59-63.

15. Souza VB, Moura Filho FJ, Souza FG, Rocha CF, Furtado FA, Goncalves TB, Vasconcelos KF: Cataract occurrence in patients treated with antipsychotic drugs. Revista brasileira de psiquiatria (Sao Paulo, Brazil : 1999) 2008, 30(3):222-226.

16. Klein BE, Klein R, Lee KE, Grady LM: Statin use and incident nuclear cataract. Jama 2006, 295(23):2752-2758.

17. Srinivasan S, Raman R, Swaminathan G, Ganesan S, Kulothungan V, Sharma T: Incidence, Progression, and Risk Factors for Cataract in Type 2 Diabetes. Investigative ophthalmology \& visual science 2017, 58(13):5921-5929.

18. Kiziltoprak H, Tekin K, Inanc M, Goker YS: Cataract in diabetes mellitus. World journal of diabetes 2019, 10(3):140-153.

19. Hendrick AM, Gibson MV, Kulshreshtha A: Diabetic Retinopathy. Primary care 2015, 42(3):451-464.

20. Blair M: Diabetes Mellitus Review. Urologic nursing 2016, 36(1):27-36.

21. Khan A, Petropoulos IN, Ponirakis G, Malik RA: Visual complications in diabetes mellitus: beyond retinopathy. Diabetic medicine : a journal of the British Diabetic Association 2017, 34(4):478-484. 
22. Haddad NM, Sun JK, Abujaber S, Schlossman DK, Silva PS: Cataract surgery and its complications in diabetic patients. Seminars in ophthalmology 2014, 29(5-6):329-337.

23. Drinkwater JJ, Davis WA, Davis TME: A systematic review of risk factors for cataract in type 2 diabetes. Diabetes/metabolism research and reviews 2019, 35(1):e3073.

24. Semeraro F, Morescalchi F, Cancarini A, Russo A, Rezzola S, Costagliola C: Diabetic retinopathy, a vascular and inflammatory disease: Therapeutic implications. Diabetes \& metabolism 2019.

25. Liew G, Wong VW, Ho IV: Mini Review: Changes in the Incidence of and Progression to Proliferative and Sight-Threatening Diabetic Retinopathy Over the Last 30 Years. Ophthalmic epidemiology 2017, 24(2):73-80.

26. Pan CW, Cheng CY, Saw SM, Wang JJ, Wong TY: Myopia and age-related cataract: a systematic review and meta-analysis. American journal of ophthalmology 2013, 156(5):1021-1033.e1021.

27. Pollreisz A, Schmidt-Erfurth U: Diabetic cataract-pathogenesis, epidemiology and treatment. Journal of ophthalmology 2010, 2010:608751.

28. Nawaz IM, Rezzola S, Cancarini A, Russo A, Costagliola C, Semeraro F, Presta M: Human vitreous in proliferative diabetic retinopathy: Characterization and translational implications. Progress in retinal and eye research 2019.

29. Liu XF, Hao JL, Xie T, Malik TH, Lu CB, Liu C, Shu C, Lu CW, Zhou DD: Nrf2 as a target for prevention of age-related and diabetic cataracts by against oxidative stress. Aging cell 2017, 16(5):934-942.

30. Wu C, Liu Z, Ma L, Pei C, Qin L, Gao N, Li J, Yin Y: MiRNAs regulate oxidative stress related genes via binding to the 3' UTR and TATA-box regions: a new hypothesis for cataract pathogenesis. BMC ophthalmology 2017, 17(1):142.

31. Ornek K, Karel F, Buyukbingol Z: May nitric oxide molecule have a role in the pathogenesis of human cataract? Experimental eye research 2003, 76(1):23-27.

32. Tan AG, Kifley A, Tham YC, Shi Y, Chee ML, Sabanayagam C, Tan NYQ, Wong KH, Mitchell P, Cumming RG et al: Six-Year Incidence of and Risk Factors for Cataract Surgery in a Multi-ethnic Asian Population: The Singapore Epidemiology of Eye Diseases Study. Ophthalmology 2018, 125(12):1844-1853.

33. Yokoyama H, Okudaira M, Otani T, Takaike H, Miura J, Saeki A, Uchigata Y, Omori Y: Existence of early-onset NIDDM Japanese demonstrating severe diabetic complications. Diabetes care 1997, 20(5):844-847.

34. Lovic D, Piperidou A, Zografou I, Grassos H, Pittaras A, Manolis A: The Growing Epidemic Of Diabetes Mellitus. Current vascular pharmacology 2019.

35. Quan J, Li TK, Pang H, Choi CH, Siu SC, Tang SY, Wat NMS, Woo J, Johnston JM, Leung GM: Diabetes incidence and prevalence in Hong Kong, China during 2006-2014. Diabetic medicine : a journal of the British Diabetic Association 2017, 34(7):902-908.

36. Xu J, Wei WB, Yuan MX, Yuan SY, Wan G, Zheng YY, Li YB, Wang S, Xu L, Fu HJ et al: Prevalence and risk factors for diabetic retinopathy: the Beijing Communities Diabetes Study 6. Retina (Philadelphia, Pa) 2012, 32(2):322-329. 
37. Shih YH, Chang HY, Lu MI, Hurng BS: Time trend of prevalence of self-reported cataract and its association with prolonged sitting in Taiwan from 2001 and 2013. BMC ophthalmology 2014, 14:128.

38. Al-Zamil WM, Yassin SA: Recent developments in age-related macular degeneration: a review. Clinical interventions in aging 2017, 12:1313-1330.

39. Tsubota K, Yokoi N, Shimazaki J, Watanabe H, Dogru M, Yamada M, Kinoshita S, Kim HM, Tchah HW, Hyon JY et al: New Perspectives on Dry Eye Definition and Diagnosis: A Consensus Report by the Asia Dry Eye Society. The ocular surface 2017, 15(1):65-76.

40. Singh S, Pardhan S, Kulothungan V, Swaminathan G, Ravichandran JS, Ganesan S, Sharma T, Raman R: The prevalence and risk factors for cataract in rural and urban India. Indian journal of ophthalmology 2019, 67(4):477-483.

\section{Abbreviations}

DM: diabetes mellitus, DR: diabetic retinopathy, NPDR: non-proliferative diabetic retinopathy, PDR: proliferative diabetic retinopathy, NHIRD: National Health Insurance Research Database, LHID: Longitudinal Health Insurance Database 2005 version, ICD-9: International Classification of Diseases, Ninth Revision, ICD-10: International Classification of Diseases, Tenth Revision, DED: dry eye diseases, AMD: age-related macular degeneration, TPPV: trans pars plana vitrectomy, Cl: confidence intervals, aHR: adjusted hazard ratios

\section{Figures}




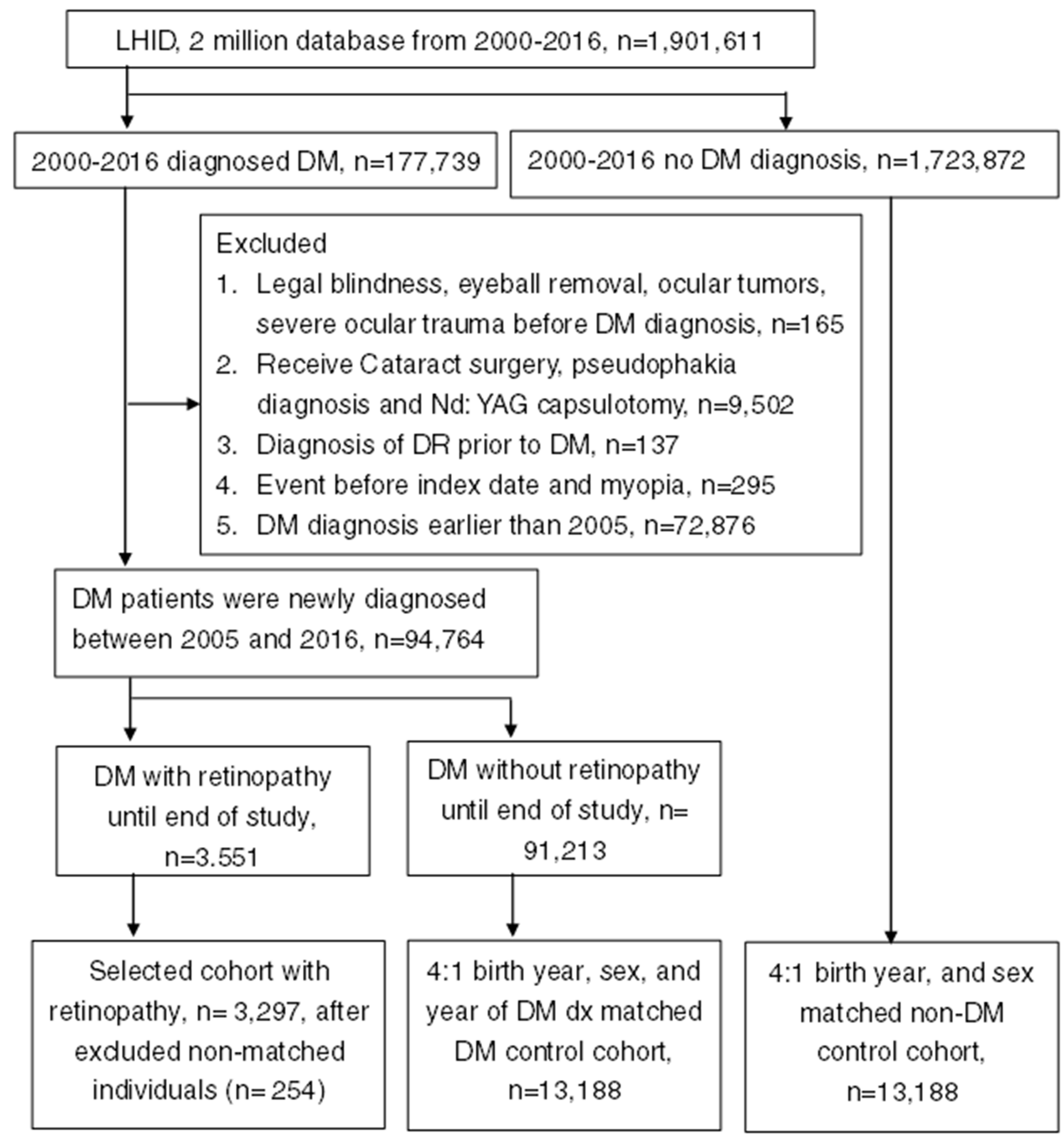

Figure 1

The flowchart of patients selection LHID: Longitudinal Health Insurance Database 2005 version DM: diabetes mellitus DR: diabetic retinopathy 


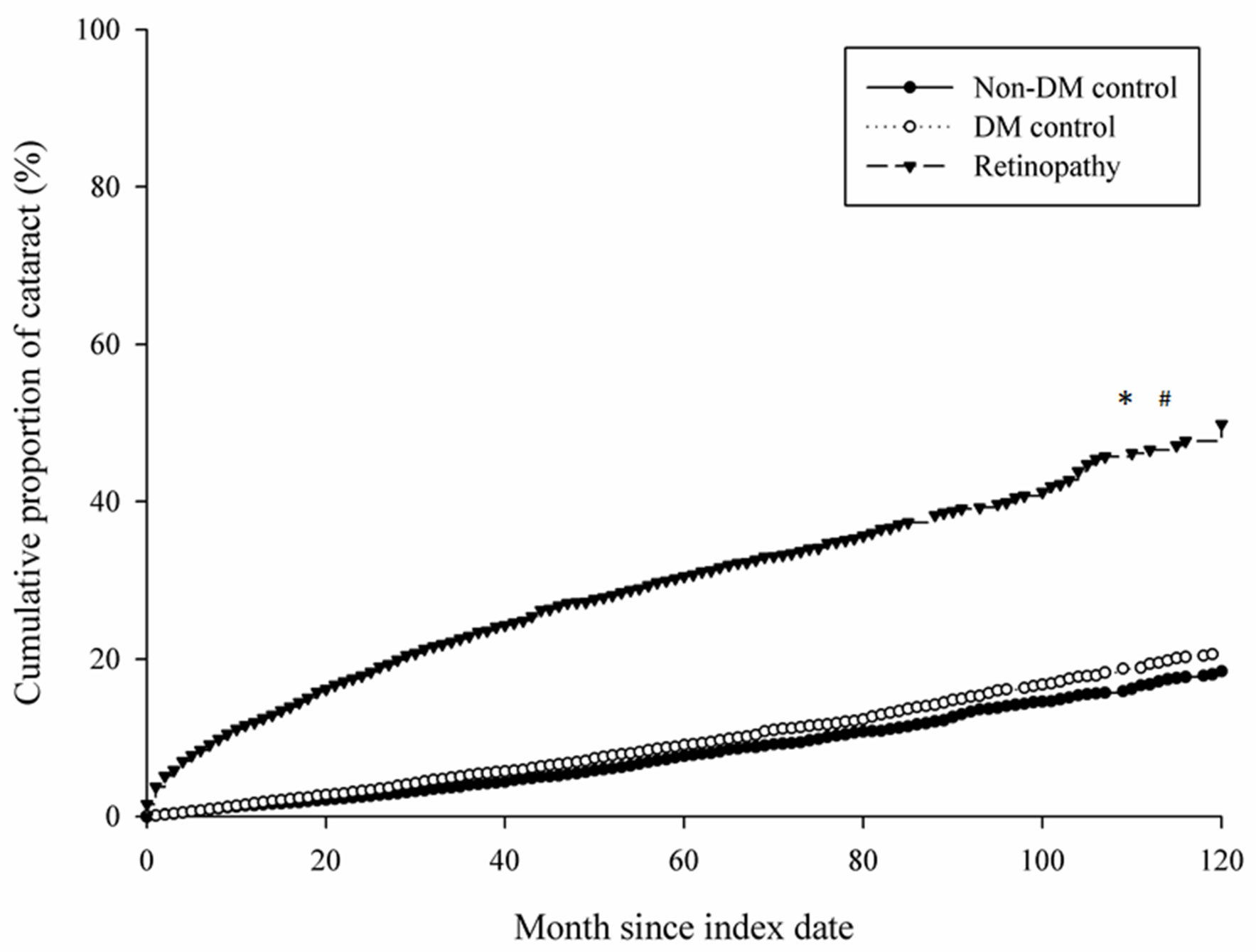

Figure 2

The cumulative probability of sight-threatening cataract among the study and control groups * denotes Log rank $P<0.001$, diabetic retinopathy versus Non-DM control \# denotes Log rank $P<0.001$, diabetic retinopathy versus DM control 